\title{
Influence of $\beta$-radiation sterilisation in properties of new chitosan/soybean protein isolate membranes for guided bone regeneration
}

\author{
R. M. SILVA ${ }^{1,2, *}$, C. ELVIRA ${ }^{3}$, J. F. MANO ${ }^{1,2}$, J. SAN ROMÁN ${ }^{3}$, R. L. REIS ${ }^{1,2}$ \\ ${ }^{1}$ 3Bs Research Group, Biomaterials, Biodegradables, Biomimetics, Campus de Gualtar 4710- \\ 057 Braga, Portugal \\ E-mail: ricardosilva@dep.uminho.pt \\ ${ }^{2}$ Department of Polymer Engineering, University of Minho, Campus de Azurém 4800-058 \\ Guimarães, Portugal \\ ${ }^{3}$ Macromolecular Chemistry Department, Instituto de Ciencia y Tecnologia de Polimeros, \\ CSIC, Juan de la Cierva 3, 28006 Madrid, Spain
}

\begin{abstract}
Novel chitosan (cts) and soybean protein isolate (SI) blended membranes were prepared. These membranes were produced by solvent casting. Besides combining the advantages of both materials, cts/SI membranes exhibit a biphasic structure that will eventually originate in situ porous formation, through a two-step degradation mechanism. In this particular work the effect of $\beta$-radiation over the properties of these membranes was evaluated. $\beta$-radiation sterilisation was performed at three different doses (25, 50 and $100 \mathrm{kGy}$ ) and eventual surface chemical changes were evaluated by Fourier transformed infrared - with attenuated total reflection and contact angle measurements. Moreover, eventual bulk properties changes due to $\beta$-radiation were assessed by means of mechanical tensile tests and water uptake measurements. In general, no substantial changes were detected on the studied properties, with the exception of the surface energy that was found to be slightly increased for higher applied doses.
\end{abstract}

(C) 2004 Kluwer Academic Publishers

\section{Introduction}

In many cases, bone healing and the formation of new bone is inhibited by the rapid appearance of connective tissue. The concept of guided bone regeneration (GBR) primarily consists in barrier membranes that prevent the in-growth of connective tissue. Furthermore, growth factors can be accumulated under the membrane $[1,2]$. One interesting approach to develop suitable barrier membranes for GBR has been focused in proteins present in the extracellular matrix, namely on using collagen [3]. However, a lot of work has still to be done to develop an ideal GBR and the recent bovine spongiform encephalopathy (BSE) crises lead to an increasing concern about the use of animal origin proteins in biomedical applications. Soybean protein isolate (SI) has been proposed as a non-animal origin protein substitute for several biomedical applications $[4,5]$. SI is a mixture of globulin proteins on which glycinin is presented at about $40 \%$ (isoelectric point - pI 6.4) and $\beta$-conglycinin at about $28 \%$ ( pI 4.8) [6]. SI is not totally soluble in water, but about $90 \%$ of the proteins present in soybean are soluble at some $\mathrm{pH}$ (water extractable) [7]. On the other hand, chitosan is a copolymer of $\mathrm{N}$-acetylglucosamine
(GlcNAc) and glucosamine (GlcN). It has been observed that GlcN, a degradation product of chitosan, has a beneficial effect on treatment and symptoms of osteoarthritis as it helps to regenerate joint cartilage $[8,9]$. Moreover, it possesses excellent properties such as biocompatibility, biodegradability and non-toxicity [10] and its degradation products are non-toxic, non-immunogenic and non-carcinogenic [11]. Chitosan has been widely studied and proposed for many biomedical applications [12-17], namely for skin tissue regeneration, wound dressings, as barrier-membranes to prevent the in-growth of undesirable connective tissue, sutures and carriers for sustained drug release [10].

Thus, blending both materials can combine the advantages of a protein material with the unique properties of chitosan. Furthermore, the organic fraction of biologically mineralised structures (like bone and tooth) are mainly composed by proteins/polysaccharide systems. Furthermore, these systems should possess different degradation behaviours as the distinct phases are degraded by different enzymes (chitosan by lysozyme $[18,19]$ and SI by non-specific proteases). So, by controlling the insoluble fraction and distribution

*Author to whom all correspondence should be addressed. 
of the protein material, which depends on factors such as $\mathrm{pH}$, mixture composition, polymer concentration, etc., it should be possible to tailor the degradation rate, leading to systems with a two-step degradation behaviour.

This work focus on the assessment of the influence of $\beta$-radiation sterilisation in the properties of the novel blended membranes composed by chitosan and SI blends. The main effects that ionising radiation exposure can eventually induce in exposed samples are, among others, crosslinking, chain scissions and oxidative processes [20]. Whenever occurring, these changes should have a remarkable effect on properties such as mechanical properties, water uptake ability and surface energy. In this work, besides the referred properties, the eventual chemical changes were analysed by Fourier transformed infrared - with attenuated total reflection (FTIR-ATR) spectroscopy.

\section{Materials and methods}

\subsection{Membranes preparation and $\beta$-radiation sterilisation}

Chitosan (deacetylation degree of about 85\%) was purchased from Sigma. SI was provided by Loders Crocklaan BV (The Netherlands). Membranes (average thickness from 45 to $65 \mu \mathrm{m}$ ) were prepared by solvent casting. Chitosan was dissolved in $1 \mathrm{wt} \%$ of acetic acid solution $(\mathrm{AcOH})$ at a concentration of $1 \mathrm{wt} \%$. SI was suspended in distilled water at room temperature under gentle stirring in order to avoid protein denaturation and consequently, foam formation. SI suspensions were added dropwise to chitosan solutions under constant stirring at different ratios (designated cts $100 \%$, cts $75 \%$, cts $50 \%$, cts $25 \%$, related to chitosan percentage), and $\mathrm{pH}$ was corrected to 4.0 with $\mathrm{AcOH}$ (equal to chitosan solution). Mixtures were poured into the moulds directly in the drying place and moulds were no longer moved or removed until complete drying, in order to assure that the insoluble part of SI was uniformly distributed. Drying was performed at room temperature (c.a. $20^{\circ} \mathrm{C}$ ) and relative humidity (c.a. 55\%). The air-exposed (AE) surface during the drying process presented some roughness at macroscopical level, whereas the mouldexposed (ME) surface presented a very smooth appearance. In this way, the surface characterisation was carried on taking in consideration this feature.

$\beta$-radiation sterilisation was preformed by Ionmed Esterilisatión, SA (Spain) at different radiation doses (25, 50 and $100 \mathrm{kGy})$ using the electron accelerator Rhodotron TT2 (10 MeV).

Both membrane surfaces were characterised morphologically by scanning electron microscopy (SEM, Philips XL30) and environmental scanning electron microscopy (ESEM, Philips XL30).

\subsection{Fourier transform infrared-with attenuated total reflection (FTIR-ATR)}

Surface chemical modifications were assessed by FTIRATR spectroscopy (Perkin-Elmer 457 Spectrometer). The oxidative effects of radiation should be detected at surface level by changes in the FTIR-ATR spectrum regions of $3000-3500$ and $1650-1800 \mathrm{~cm}^{-1}$ [21]. This spectrum regions were analysed in more detail for chemical shifts, peak shape and intensity changes. AE and ME surfaces were analysed.

\subsection{Contact angle measurements}

Contact angle $(\theta)$ measurements were undertaken by means of sessile drop method with contact angle measurement system G10 from Krüss at room temperature (c.a. $20^{\circ} \mathrm{C}$ ). At least five measurements were performed for each solvent. Surface tension $\left(\sigma_{s}\right)$, as well as its polar $\left(\sigma_{s}^{p}\right)$ and dispersive $\left(\sigma_{s}^{d}\right)$ components were determined by Owens and Wendt method [22], using the equipment software G402. Glycerol and methylene iodide were respectively used as polar and non-polar test liquids. Both $\mathrm{AE}$ and $\mathrm{ME}$ surfaces were tested.

\subsection{Water uptake measurements}

The water uptake measurements were carried out by means of immersing previously weighed $\left(M_{0}\right)$ samples,

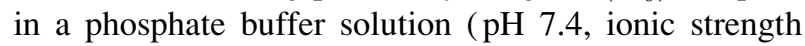
$0.154 \mathrm{M}$, buffer concentration $50 \mathrm{mM}$ ) at $37^{\circ} \mathrm{C}$. Containers were sealed and placed in a thermostatic bath at $37 \pm 1{ }^{\circ} \mathrm{C}$. For each condition four samples were used. After each time period (from $10 \mathrm{~s}$ to $48 \mathrm{~h}$ ) samples were removed from containers, adsorbed water was removed by sandwiching between two paper towels and weighed immediately $(M)$. The water uptake $(W)$ was calculated using the following equation:

$$
W=\frac{M-M_{0}}{M_{0}}=\frac{M}{M_{0}}-1
$$

\subsection{Quasi-static mechanical properties}

Membranes were cut into strips. Their dimensions were found to be typically about $8 \times 1 \times 0.045 \mathrm{~mm}$. Thickness was taken as a mean of 10 values at different points measured with a low-pressure micrometre. Their resistance to stretching was evaluated on a Perkin-Elmer DMA7e at a constant stress rate of $5 \mathrm{MPa} / \mathrm{min}$ using the tensile mode. In such experiments, the strain was monitored as a function of stress. Please note that such procedure is different from conventional mechanical tests where the stress is monitored as a function of strain, which varies at constant rate. However, one can also build stress-strain curves and obtain a measure of the stiffness (by looking at the slope of the curve at early stages) and the strength (measured by the stress at break) of the sample, when experiments at constant stress rate are performed. Mechanical tests were carried out at room temperature (c.a. $20^{\circ} \mathrm{C}$ ) and relative humidity (c.a. $70 \%$ ). Secant modulus was calculated at $1 \%$ of elongation. Stress and strain at break were also estimated.

\subsection{Density determination}

The polymer blends density measurements were undertaken making use of $n$-heptane/dibromoethane soluble solvents system. The rational for the solvents choice was 
based on their density (polymer density limited by solvents density) and the fact that samples did not absorb any measurable quantity of both. Membranes were cut in at least three strips (c.a. $1 \times 20 \mathrm{~mm}$ ). A test tube was filled with $n$-heptane and placed in a ultrasound bath for 5 min to eliminate air bubbles (at this stage membrane strips should be settled down at the bottom). Then, dibromoethane was added drop by drop until all polymer strips start to go up and tend to float. At this stage the liquid density, determined by pycnometry, should be an approximation of the polymer density. The procedure was repeated three times giving results with a good precision (maximum standard deviation of 0.0058). In general, measures revealed to be accurate since at the changing point the addition of a few drops did not change significantly the liquid density.

\section{Results and discussion}

\subsection{FTIR-ATR}

FTIR analysis was applied in order to detect chemical modifications on irradiated samples by means of oxidation or crosslinking processes. The most representative signals (see Fig. 1) are those assigned in the $3400-3200 \mathrm{~cm}^{-1}$ interval corresponding to the stretching vibration of the hydroxyl groups, $\mathrm{NH}_{2}$ and $-\mathrm{NH}-$ of chitosan, and to the NH groups of amines and amides of the amino-acids (SI). Those signals appearing at $1626 \mathrm{~cm}^{-1}$, which can be assigned to the $\mathrm{NH}_{2}$ bending vibration (chitosan), amide vibration (SI), and signals appearing at $1530 \mathrm{~cm}^{-1}$ to the aromatic ring of some amino acids of SI. In terms of different radiation doses no significant changes are observed in the corresponding signal also in comparison to non-irradiated samples, indicating that significant chemical modifications are not observed by this spectroscopic technique when treating cts/SI membranes with $\beta$-radiation under the mentioned conditions.

\subsection{Contact angle measurements}

Contact angle measurements were performed on all treated samples in order to determine the surface energy, the respective polar and dispersive contribution, and the

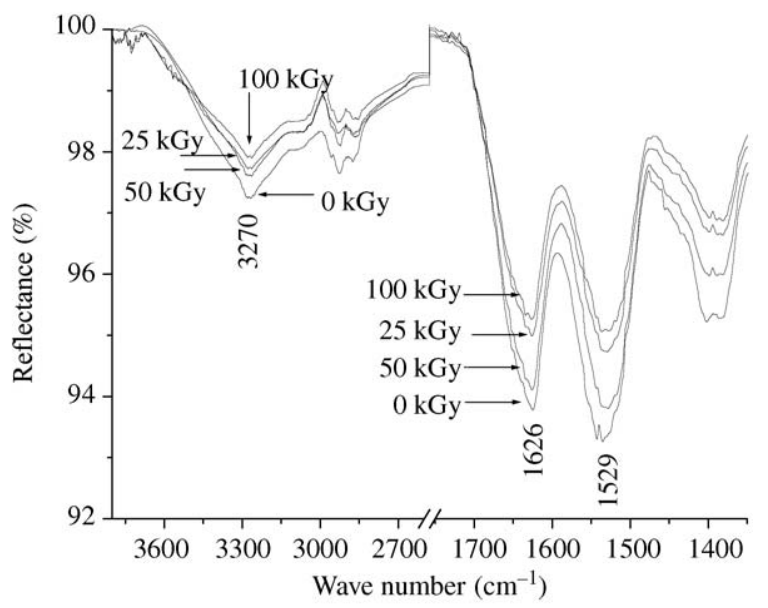

Figure 1 FTIR-ATR spectra of the ME surface of cts $75 \%$ membranes treated with different doses of $\beta$-radiation. Scale was adjusted to see in detail $3000-3500$ and $1650-1800 \mathrm{~cm}^{-1}$ spectra regions. possible changes due to the $\beta$-radiation treatment. The contribution of the dispersive $\left(\sigma_{d}\right)$ and polar interactions $\left(\sigma_{p}\right)$ to the surface energy was calculated by considering that the intermolecular attraction, which causes surface free energy $(\sigma)$ results from a variety of intermolecular forces according to the additive rule. Thus, surface energy and contact angle of a liquid on a solid were calculated as described in the experimental part. Fig. 2 shows the total surface energies and the respective polar and dispersive components values versus the $\beta$-radiation doses in the different cts/SI formulations. From this figure it can be observed that as the SI percentage increases in the blends composition the surface energy also increases as do both components, polar and dispersive. The $\sigma_{d}$ values are plotted in Fig. 2(a), where it can be observed an increase in the dispersive energy component when increasing the amount of $\beta$-radiation doses in formulations like cts $25 \%$, cts $50 \%$, cts $100 \%$, whereas in the case of cts $75 \% \sigma_{d}$ is maintained constant. A similar behaviour is observed in the polar component energy values (Fig. 2(b)) in cts $25 \%$, cts $50 \%$ and cts $75 \%$, whereas in cts $100 \%$ the $\sigma_{p}$ decreases with the number of $\beta$-radiation doses. Finally, the addition of both compo-
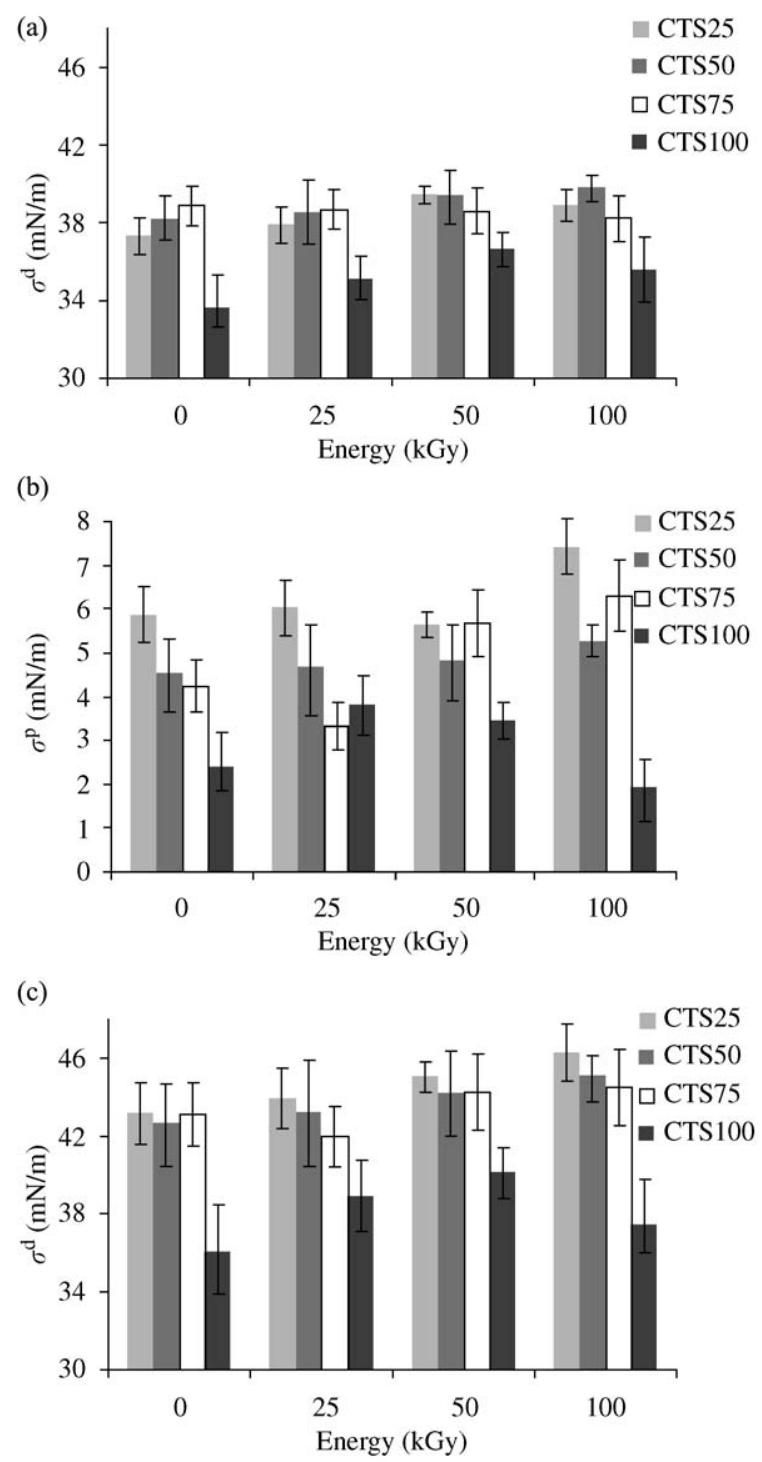

Figure 2 Dispersive (a) and polar (b) components of surface energy (c) measured at the ME surface in function of applied $\beta$-radiation dose. Data represents mean \pm error at $95 \%$ of confidence level. 

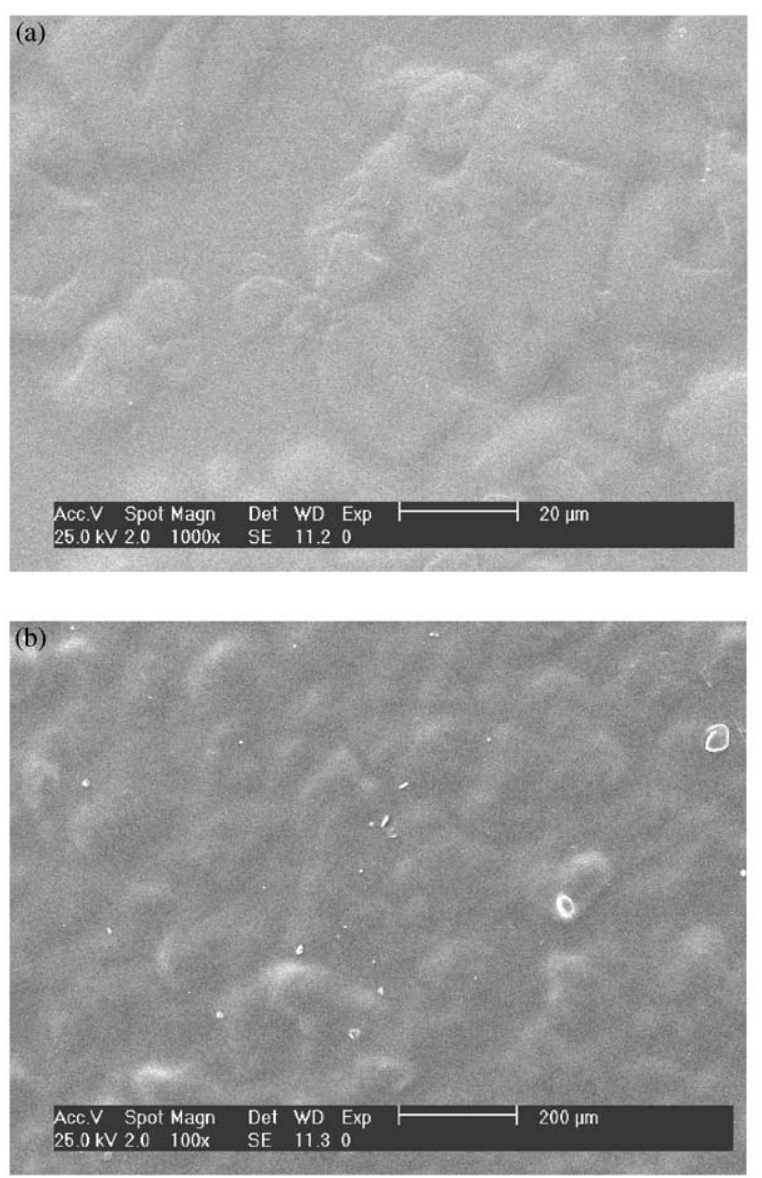

Figure 3 SEM micrographs of cts $75 \%$ non-irradiated membranes: (a) ME surface $(1000 \times)($ b) $\mathrm{AE}$ surface $(100 \times)$.

nents to obtain the final surface energy (see Fig. 2(c)) shows a general increase tendency, in about $2 \mathrm{mN} / \mathrm{m}$ units of energy, with respect to non-treated samples.

\subsection{Morphological characterisation by SEM and ESEM}

The AE surface exhibited a granulate aspect, in contrast with the smooth ME surface for all cts/SI compositions, by simple eye observation. Reversely, AE surfaces present a smooth appearance at higher magnifications and it was possible to observe in the ME surface the presence of some globular structures incorporated in a continuous matrix, providing roughness at a lower scale (see Figs. 3 and 4). This seems to indicate, in the authors' view, that during the drying time insoluble suspended SI particles should settle down at the bottom. Therefore, bottom membrane surface can possibly present some SI insoluble particles, which are not totally covered by a chitosan layer. On the contrary, in the upper surface a chitosan layer should cover totally SI insoluble particles and accompany the gaps left by SI particles, originating the final rough appearance. Moreover, cts $100 \%$ membranes present both surfaces rather smooth at all magnifications used (results not shown). When comparing the blends membrane structure with the ones of the cts $100 \%$, one can correlate such granules with the SI insoluble part. This assumption is in a certain way supported by the fact that such granules present a higher swelling with respect to the surrounding matrix as it was possible to detect by ESEM (Fig. 4), being SI more
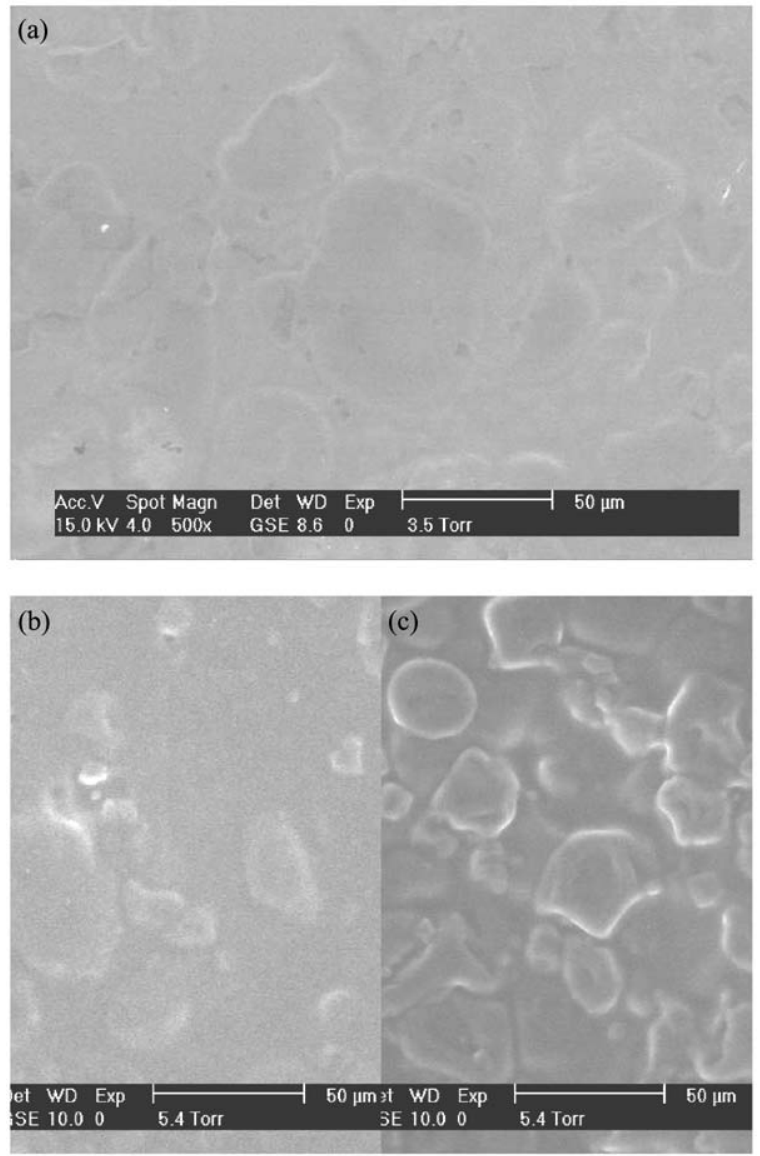

Figure 4 ESEM micrographs of cts $75 \%$ non-irradiated membranes in dry state $(660 \mathrm{~Pa})$ (a) and previously swollen in a buffer solutions at $\mathrm{pH}$ of 7.4 (b) and at 6.5 (c).

hydrophilic than chitosan. Any change in the membrane morphology was not detected after being sterilised by $\beta$ radiation at the tested doses.

\subsection{Swelling kinetics}

Fig. 5(a) and (b) show two examples of the typical variation of the water uptake as a function of time. All formulations were found to reach the maximum of the hydration degree in less than $5 \mathrm{~min}$. After that, a decrease in the water uptake was observed towards the hydration equilibrium degree. This effect was more accentuated for the formulations with superior chitosan content, being probably due to the $\mathrm{pH}$ equilibration process inside the polymeric matrix. The hydration equilibrium degree values were taken after two days of immersion and it is shown to be independent of the $\beta$-radiation treatment. In respect to the cts/SI blend composition no considerable differences could be observed (see Fig. 5(c)).

\subsection{Quasi-static mechanical properties}

Tensile tests were carried out in order to evaluate the impact of $\beta$-radiation on the mechanical performance of the several prepared formulations, since if crosslinking reactions and/or polymer oxidative degradation/depolymerisation had occurred, it would cause important changes on those properties. It was impossible to test membranes with higher SI content, because of its high brittleness. In Fig. 6(a) and (b), is represented the tensile 

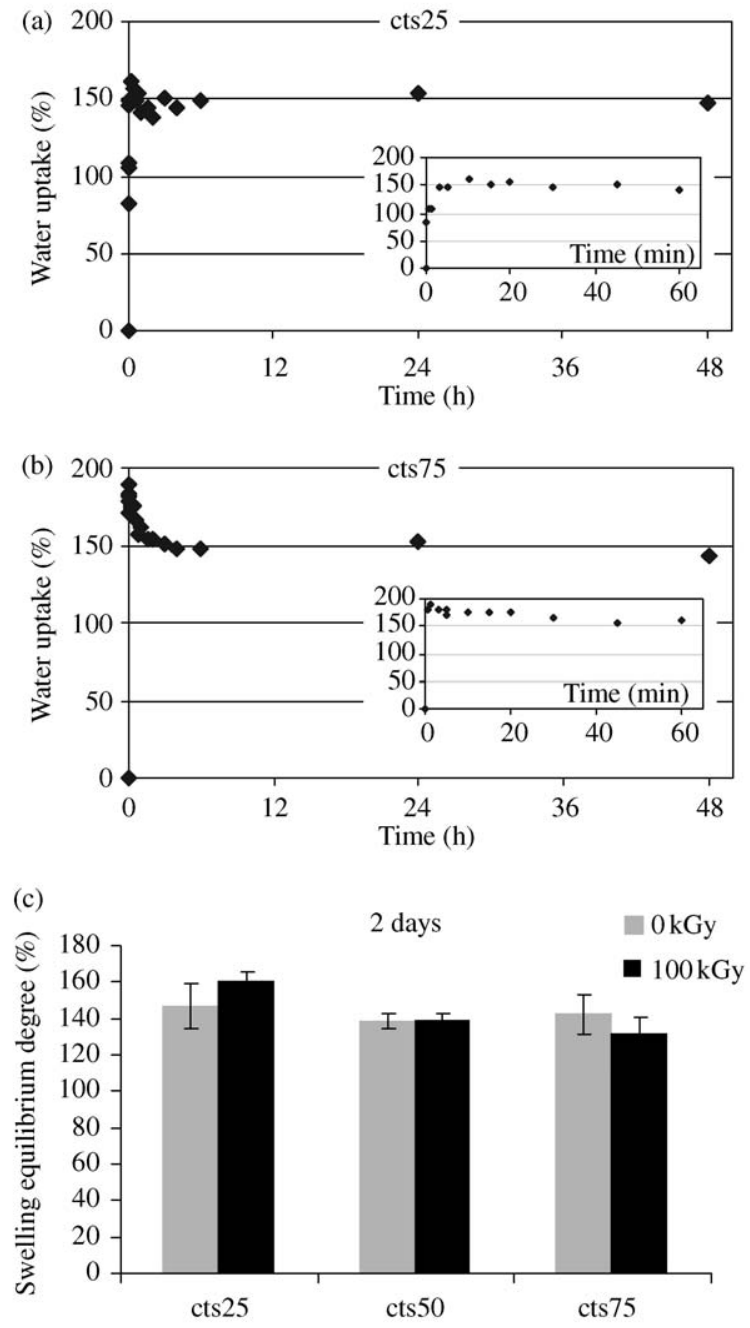

Figure 5 Swelling kinetics profile of non-irradiated cts $25 \%$ (a) and cts75\% (b) and the equilibrium hydration degree taken at two days of immersion in buffer solution ( $\mathrm{pH} 7.4$; IS $0.154 \mathrm{M}$; buffer conc. $50 \mathrm{mM}$ ) as a function of chitosan percentage in the blends for non-treated and exposed to $\beta$-radiation ( $100 \mathrm{kGy})$ membranes (c). Data represents mean \pm standard deviation of at least three samples.

strength and the secant modulus, respectively, as a function of the applied $\beta$-radiation dose. From those figures it can be observed that $\beta$-radiation did not affect the strength and the stiffness of the tested formulations, with the exception of cts50\% where the strength and the stiffness were found to increase when applying a $\beta$ radiation dose of $100 \mathrm{kGy}$. On the other hand, chitosan membranes (cts100\%) presented higher values of tensile strength at break (64.7 MPa) and modulus (2.5 GPa), than the blends. Furthermore, the amount of SI among the studied compositions did not change substantially these properties, which was found to be around $45 \mathrm{MPa}$ and 2.0 GPa, respectively. The same behaviour was also observed for the strain at break (Fig. 6(c)), being about $12 \%$ for chitosan membranes (cts100\%) and 3-4\% for the blends. However, the brittleness did not vary consistently with the $\beta$-radiation treatment, even when applying a $100 \mathrm{kGy} \beta$-radiation dose.

\subsection{Polymer density}

Polymer density was determined in order to calculate the medium average molecular weight between possible
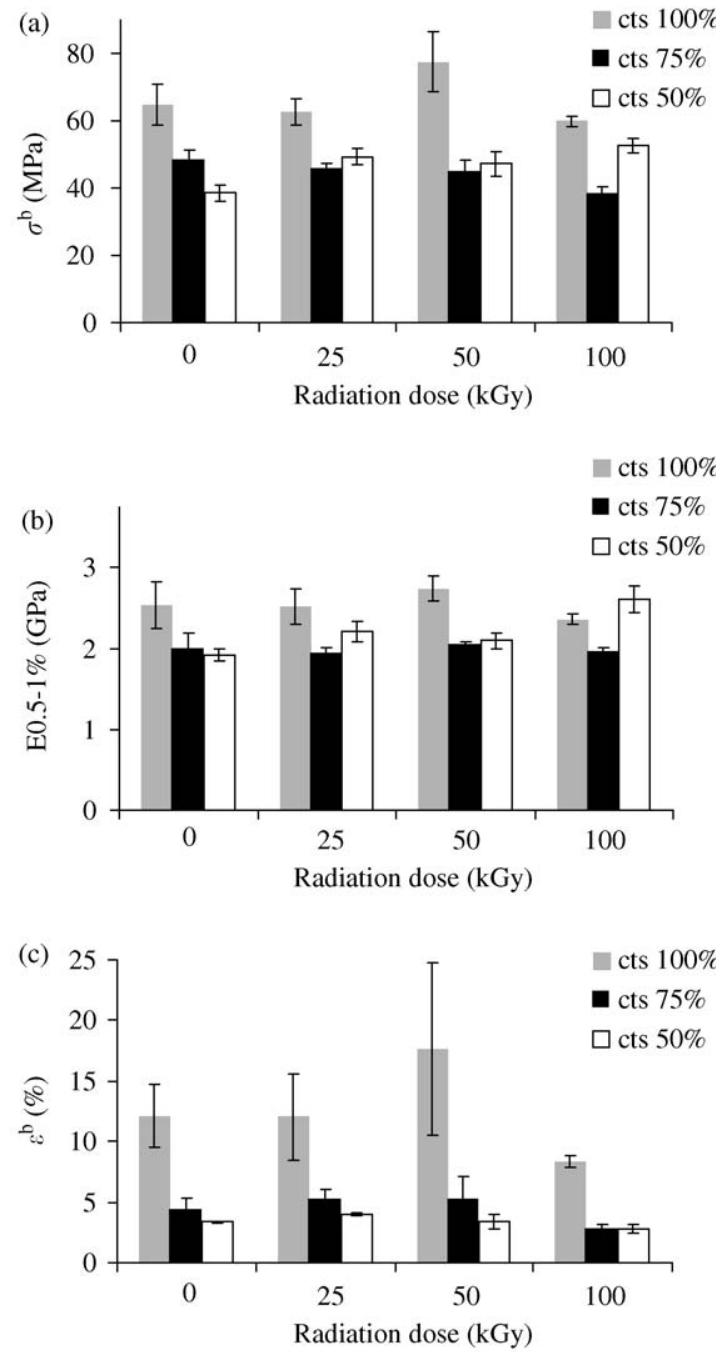

Figure 6 Membranes tensile properties of several cts/SI blend compositions as a function of applied $\beta$-radiation dose: (a) stress at break; (b) secant modulus at $2 \%$ of elongation; (c) strain at break. Data represents mean \pm standard deviation of at least three experiments.

T A B L E I Polymer density as a function of chitosan percentage in the blends for non-treated and exposed to $\beta$-radiation (100 kGy) membranes. Data represents mean \pm error at $95 \%$ of confidence level

\begin{tabular}{lll}
\hline Sample radiation dose (kGy) & 0 & 100 \\
\hline cts100\% & $1.420 \pm 0.025$ & $1.424 \pm 0.016$ \\
$\operatorname{cts} 75 \%$ & $1.386 \pm 0.017$ & $1.380 \pm 0.010$ \\
$\operatorname{cts} 50 \%$ & $1.334 \pm 0.008$ & $1.355 \pm 0.018$ \\
$\operatorname{cts} 25 \%$ & $1.314 \pm 0.009$ & $1.332 \pm 0.005$ \\
\hline
\end{tabular}

crosslinks on irradiated samples. However, since the water uptake and the modulus of all formulation were not substantially affected by the $\beta$-radiation treatment, it is presented as a further indication that no remarkable crosslinking reactions are taking place (see Table I).

\section{Conclusions}

Membranes presenting a very interesting morphology and correspondent properties could be obtained from combining chitosan and SI on blended membranes. The 
partial insolubility of $\mathrm{SI}$ at the processing $\mathrm{pH}$, as well as its asymmetric distribution through the transversal section, being the SI insoluble particles concentrated at the ME surface, are desirable features to attain a controlled degradation rate in vivo. Furthermore, it can be foreseen a two-step degradation mechanism, eventually leading to in situ porous formation, which might be clinically useful. Moreover, since in general no remarkable differences were observed for the studied bulk and surface properties of the membranes, it might be possible to tailor their degradation and their biological response without changing their key properties, by means of controlling blends' composition.

$\beta$-radiation seems to be a suitable sterilisation methodology to be used on chitosan/SI membranes aiming to be used in GBR. In fact, no considerable changes could be detected on the mechanical properties and equilibrium hydration degree. Furthermore, FTIRATR analyses indicated that no substantial chemical modifications were occurring when sterilising samples by $\beta$-radiation. The most sensitive property to $\beta$ radiation exposure was the surface energy. In fact, a slight increase tendency was observed for the surface energy due to $\beta$-radiation.

\section{Acknowledgments}

R. M. Silva was supported by the Ph.D. Scholarship SFRH/BD/6862/2001 from the Portuguese Foundation for Science and Technology (FCT) under the POCTI programme. This work was partially supported by FCT through funds from the POCTI and/or FEDER programmes. Ionmed Esterelisatión, SA is also acknowledged by performing $\beta$-radiation sterilisation.

\section{References}

1. Y. UEYAMA, K. ISHIKAWA, T. MANO, T. KOYAMA, H. NAGATSUKA, K. SUZUKI and K. RYOKE, Biomaterials 23 (2002) 2027.

2. A. V. imbronito, J. H. TOdescan, C. V. CARVAlho and V. E. ARANA-CHAVEZ, ibid. 23 (2002) 4079.

3. H. JIANQI, H. HONG, S. LIEPING and G. GENGHUA, J. Oral Maxillofac. Surg. 60 (2002) 1449.

4. C. M. VAZ, L. A. DE GRAAF, R. L. REIS and A. M. CUNHA, Polym. Degrad. Stabil. 81 (2003) 65.

5. C. M. VAZ, P. F. N. M. VAN DOEVEREN, R. L. REIS and A. M. CUNHA, Polymer 44 (2003) 5983.

6. M. C. GARCIA, M. TORRE, F. LABORDA and M. L. MARINA, J. Chromatogr. A 758 (1997) 75.

7. J. M. S. RENKEMA, C. M. M. LAKEMOND, H. H. J. DE JONGH, H. GRUPPEN and T. VAN VLIET, J. Biotechnol. 79 (2000) 223.

8. J. I. FENTON, K. A. CHLEBEK-BROWN, T. L. PETERS, J. P. CARON and M. W. ORTH, Osteoarthr. Cartil. 8 (2000) 444.

9. J. I. FENTON, K. A. CHLEBEK-BROWN, T. L. PETERS, J. P. CARON and M. W. ORTH, ibid. 8 (2000) 258.

10. M. N. V. RAVI KUMAR, React. Funct. Polym. 46 (2000) 1.

11. R. A. A. MuZzarelli, Carbohydr. Polym. 20 (1993) 7.

12. M. ITO, Y. HIDAKA, M. NAKAJIMA, H. YAGASAKI and A. H. KAFR AW Y, J. Biomed. Mater. Res. 45 (1999) 204.

13. D. THACHARODI and K. P. RAO, Biomaterials 16 (1995) 145.

14. F.-L. MI, Y.-C. TAN, H.-F. LIANG and H.-W. SUNG, ibid. 23 (2002) 181.

15. J. MA, H. WANG, B. HE and J. CHEN, ibid. 22 (2001) 331.

16. M. TAKECHI, K. ISHIKAWA, Y. MiYAmoto, M. NAGAYAMA and K. SUZUKI, J. Mater. Sci.-Mater. Med. 12 (2001) 597.

17. M. PRASITSILP, R. JENWITHISUK, K. KONGSUWAN, N. DAMRONGCHAI and P. WATTS, ibid. 11 (2000) 773.

18. K. KURITA, Y. KAJI, T. MORI and Y. NISHIYAMA, Carbohydr. Polym. 42 (2000) 19.

19. K. томінAтA and Y. IK A DA, Biomaterials 18 (1997) 567.

20. J. Silverman, Radiat. Phys. Chem. 9 (1997) 1.

21. S. BaCCAro and U. Buontempo, Int. J. Radiat. Appl. Instrum. Part C. Radiat. Phys. Chem. 40 (1992) 175.

22. D. K. OWENS and R. C. WENDT, J. Appl. Polym. Sci. 13 (1969) 1741 .

Received 4 October

and accepted 10 October 2003 